\title{
Correction to: Foraging Profile, Activity Budget and Spatial Ecology of Exclusively Natural-Foraging Chacma Baboons (Papio ursinus) on the Cape Peninsula, South Africa
}

\author{
Matthew C. Lewis $^{1}$ • M. Justin O'Riain ${ }^{1}$
}

Published online: 26 February 2018

(C) Springer Science+Business Media, LLC, part of Springer Nature 2018

\section{Correction to: Int J Primatol https://doi.org/10.1007/s10764-017-9978-5}

The original version of this article unfortunately contained two mistakes in captions in the "Activity Budgets" section. The relevant figure and table, with the correct captions (corrections underlined), are shown below.

Both mistakes were made by the authors in the final submission and were not noticed at proofing stage.

The online version of the original article can be found at https://doi.org/10.1007/s10764-017-9978-5

Matthew C. Lewis

matt.lewis.uct@gmail.com

1 Department of Biological Sciences, University of Cape Town, Private Bag X3, Rhodes Gift, Cape Town 7701, South Africa 
a

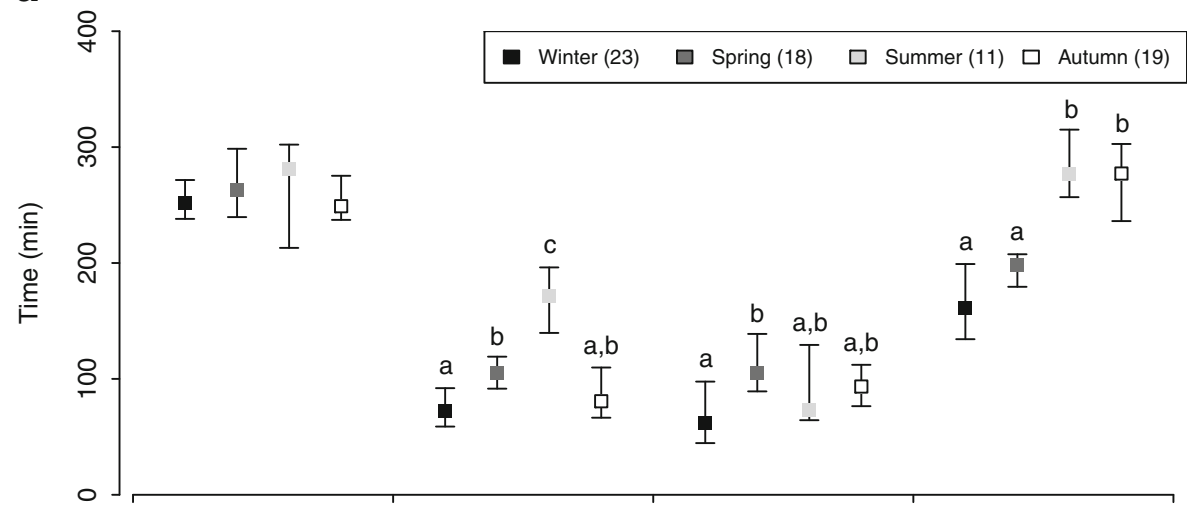

b

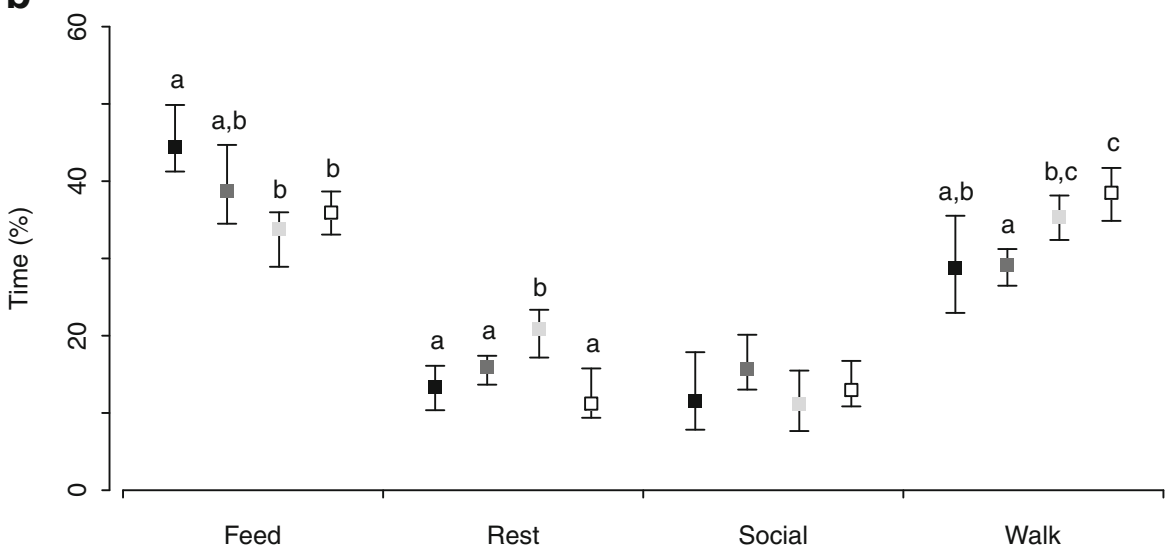

Activity

Fig. 3 Activity budgets of a troop of natural-foraging chacma baboons (Papio ursinus) on the Cape Peninsula, South Africa, divided into times (in minutes) allocated to different activities (a), and percentages of daylight hours allocated to different activities (b). Values were derived from data collected through instantaneous scan sampling between June 1, 2009 and June 27, 2010. Values in parentheses in the legend indicate numbers of full days per season; points indicate median values and error bars denote inter-quartile ranges; lower case letters in plots indicate significant differences (values that share letters are not significantly different) 
Table IV Results of Wilcoxon's rank sum tests for differences in time allocated to different activities across seasons by a group of natural-foraging chacma baboons (Papio ursinus) on the Cape Peninsula, South Africa, between June 1, 2009 and June 27, 2010

\begin{tabular}{|c|c|c|c|c|c|}
\hline \multirow[b]{2}{*}{ Food category } & \multirow[b]{2}{*}{ Seasons } & \multicolumn{2}{|c|}{ Time (min) } & \multicolumn{2}{|c|}{ Time (\% daylight hours) } \\
\hline & & $W$ & $P_{\text {Holm }}$ & $W$ & $P_{\text {Holm }}$ \\
\hline \multirow[t]{6}{*}{ Feeding } & winter-spring & - & - & 135 & 0.179 \\
\hline & winter-summer & - & - & 28 & $<0.001$ \\
\hline & winter-autumn & - & - & 63 & $<0.001$ \\
\hline & spring-summer & - & - & 147 & 0.124 \\
\hline & spring-autumn & - & - & 120 & 0.252 \\
\hline & summer-autumn & - & - & 133 & 0.252 \\
\hline \multirow[t]{6}{*}{ Resting } & winter-spring & 317 & 0.010 & 256 & 0.410 \\
\hline & winter-summer & 247 & $<0.001$ & 220 & 0.001 \\
\hline & winter-autumn & 262 & 0.306 & 193 & 0.532 \\
\hline & spring-summer & 16 & $<0.001$ & 35 & 0.013 \\
\hline & spring-autumn & 124 & 0.306 & 114 & 0.258 \\
\hline & summer-autumn & 17 & $<0.001$ & 24 & 0.001 \\
\hline \multirow[t]{6}{*}{ Social activity } & winter-spring & 309 & 0.040 & - & - \\
\hline & winter-summer & 161 & 0.860 & - & - \\
\hline & winter-autumn & 299 & 0.220 & - & - \\
\hline & spring-summer & 123 & 0.880 & - & - \\
\hline & spring-autumn & 136 & 0.880 & - & - \\
\hline & summer-autumn & 120 & 0.880 & - & - \\
\hline \multirow[t]{6}{*}{ Travel } & winter-spring & 286 & 0.081 & 217 & 0.805 \\
\hline & winter-summer & 242 & $<0.001$ & 181 & 0.137 \\
\hline & winter-autumn & 400 & $<0.001$ & 345 & 0.005 \\
\hline & spring-summer & 13 & $<0.001$ & 42 & 0.038 \\
\hline & spring-autumn & 298 & $<0.001$ & 291 & $<0.001$ \\
\hline & summer-autumn & 85 & 0.420 & 141 & 0.245 \\
\hline
\end{tabular}

$N_{\text {winter }}=23, N_{\text {spring }}=18, N_{\text {summer }}=11, N_{\text {autumn }}=19$; bold font denotes significance at the $5 \%$ significance level based on $P$-values with a Holm correction applied 